Original Article - Clinical Science

\title{
Fungal endophthalmitis: A 20-year experience at a tertiary referral centre
}

\author{
Gursimrat K. Bhullar MBBS MMed(OphthSc), 1,2 Rosie C.H. Dawkins MPH \\ FRANZCO, ${ }^{1,2,3}$ Rodger A. Paul BSc(Hons) ${ }^{1}$ and Penelope J. Allen FRANZCO $1,2,3$ \\ 1. The Royal Victorian Eye and Ear Hospital, Melbourne, Victoria, Australia \\ 2. Centre for Eye Research Australia, Melbourne, Victoria, Australia \\ 3. The University of Melbourne, Melbourne, Victoria, Australia
}

Correspondence: A/Prof. Penelope J Allen, Royal Victorian Eye and Ear Hospital, 32 Gisborne Street, East Melbourne, Victoria 3002 Australia

Email: pjallen@melbourneretina.com.au

Short running title: Fungal endophthalmitis

Received 7 February 2020; accepted 28 J une 2020

Funding sources / Financial disclosure: Dr Rosie Dawkins holds a RVEEH Early Research Career Support Grant.

\section{Conflict of interest: None}

Early findings of this research were presented at the APVRS Congress in Shanghai in November 2019 - awarded "First Prize - Poster Category".

This is the author manuscript accepted for publication and has undergone full peer review but has not been through the copyediting, typesetting, pagination and proofreading process, which may lead to differences between this version and the Version of Record. Please cite this article as doi: $10.1111 /$ ceo.13820

This article is protected by copyright. All rights reserved. 


\section{ABSTRACT}

I mportance: Fungal endophthalmitis is an uncommon and serious intraocular infection, often with poor outcomes. This study examines the trend in this disease over twenty years, to inform clinician decision-making and optimise patient outcomes.

Background: Due to infrequent presentation of fungal endophthalmitis, there is limited understanding to formulate a standardised approach to management.

Design: A prospective case series over the period January 1, 1999 to December 31, 2018.

Participants: Patients with clinically diagnosed fungal endophthalmitis managed at the Royal Victorian Eye and Ear Hospital, Melbourne, Australia.

Methods: Review of the Victorian Endophthalmitis Registry for endophthalmitis episode of each patient.

Main outcome measures: Patient demographics, co-morbidities, visual acuity at presentation, aetiology, treatment, microbiology results and final visual acuity outcome.

Results: Eighty-four cases of fungal endophthalmitis were identified over the study period with a median age of 43.5 years [IQR $30.8-63.0$ ]. $65.5 \% \quad(n=55)$ of patients were male. $81.0 \%(n=68)$ of these cases were secondary to endogenous causes, of which $55.9 \%$ were associated with intravenous drug use (IVDU). Among the exogenous causes, penetrating eye injury (56.3\%) was the most common aetiological factor. 39 patients (46.4\%) grew Candida species from ocular fluid specimens, all of which were sensitive to fluconazole. 
Conclusion and relevance: Our case series provides important insights into fungal endophthalmitis - a high degree of suspicion for fungal endophthalmitis in patients with history of IVDU, and relatively good outlook for vision when Candida is the causative organism. This should allow institutions to implement a standardised management strategy based on evidence.

Keywords: Endophthalmitis, Fungal, Endogenous endophthalmitis

\section{I NTRODUCTI ON}

Fungal endophthalmitis persists as a devastating and uncommon form of endophthalmitis with poor clinical outcomes, both visual and structural. The aetiology of this intraocular infection can be divided into exogenous and endogenous sources. While endogenous endophthalmitis is secondary to haematogenous spread of the fungal pathogen to the eye via the blood-ocular barrier from distant sites of the body, exogenous tends to be direct invasion of the microorganism secondary to keratitis, trauma, or intraocular surgery. Both types of endophthalmitis can cause devastating structural effects to the eye leading to visual loss. ${ }^{1,2,3}$

Risk factors for endogenous fungal endophthalmitis include recent hospitalisation, diabetes mellitus, immunosuppression, urinary tract infection, intravenous drug use (IVDU), and use of indwelling urinary catheters. ${ }^{4}$ It may however occur in immunocompetent and healthy individuals., 5,7 Due to Australia's ageing population, advances in medicine and recreational drug abuse, there has been a steady increase in the incidence of fungal endophthalmitis. ${ }^{8}$ This disease burden adds to an existing high prevalence of cases of fungal endophthalmitis in developing countries. ${ }^{8,9,10}$ 
Yeasts have been identified as the most common causative organism of endogenous fungal endophthalmitis in the literature, ${ }^{11,12,13}$ whilst the organisms are variable and dependent on the causative mechanism in an exogenous source. ${ }^{14}$ The offending pathogens vary geographically with high prevalence of both Candida spp. and Aspergillus spp. Both of these cause a progressive infection and are associated with a poorer visual prognosis if not managed appropriately. ${ }^{13,15,16}$

Better visual outcomes have been seen in cases with fungal disease when compared to bacterial endophthalmitis. ${ }^{11}$ Prompt clinical diagnosis and timely empirical treatment is strongly advised to preserve structural integrity and visual outcomes in all cases of fungal endophthalmitis. An intravitreal antifungal agent and/or systemic fungal therapy can be utilised. The role of vitrectomy in fungal endophthalmitis is controversial and not fully understood but may play a role at least for diagnostic purposes. ${ }^{2,11}$

Currently published data is limited in its spectrum of fungal disease and its management, particularly in the Australian context. The challenge persists due to the difficulty in diagnosis and limited curative options in some situations. The purpose of this study is to capture the trend of fungal endophthalmitis at a tertiary referral centre over twenty years to aid clinicians with the changes seen in presentation and management of these fungal endophthalmitis cases. The incidence, presentation, visual outcomes, microbiological profile and management have been outlined for all cases with suspected fungal endophthalmitis.

\section{METHODS}

The Victorian Endophthalmitis Registry is prospectively maintained at the Royal Victorian Eye and Ear Hospital (RVEEH), Melbourne, Australia by one of the authors 
(PJ A). This prospective database was initiated in 1998 and includes all cases of endophthalmitis presenting to the RVEEH.

All fungal endophthalmitis cases during the period of J anuary 1, 1999 to December 31, 2018 were included in this study. This was defined as all cases with proven fungal growth on microbiology analysis or those with features of fungal infection seen on clinical ophthalmic examination, that is, anterior/posterior segment inflammation, vitritis or characteristic fundal lesions. Suspected cases treated for fungal infection without an organism growth also had a supporting history of endogenous source (e.g. history of IVDU), penetrating eye injury (PEI) or delayed presentation of postoperative endophthalmitis.

Supporting data collected within the registry includes demographic information, affected eye (including the side of more severe disease if there was bilateral involvement), possible aetiology and risk factors, visual acuity (VA) on presentation and at 3-month (or most recent) follow - up appointment. The research was conducted in accordance with the principles of Declaration of Helsinki and local hospital ethics committee guidelines.

All microbiological analysis of intraocular fluid (aqueous and/or vitreous) samples was carried out at St. Vincent's Hospital, Melbourne, Australia. The treating clinician acquired these samples in accordance to hospital protocol using an aseptic technique. Pathology results were reviewed for intraocular samples tested, organism growth and sensitivities.

The details of the treatment were also recorded - intravitreal and/or systemic antifungal therapy, early ( $<24$ hours) or delayed ( $>24$ hours) vitrectomy, and further surgical procedures. Presenting Snellen visual acuity (VA) was grouped into 
six categories - no vision impairment (better than 6/12), mild vision impairment (6/12 to $6 / 18$ ), moderate vision impairment (worse than $6 / 18$ to $6 / 60$ ), severe vision impairment (worse than $6 / 60$ to $3 / 60$ ), blind (worse than $3 / 60$ including no light perception) as per ICD-11 diagnosis coding. ${ }^{17}$ Visual outcomes were defined as improved if the Snellen VA gained by two or more rows and in cases with 'beyond Snellen' acuity of hand movements (HM), count fingers (CF), perception to light (PL) or no perception to light (NPL); it improved by one or more steps.

Descriptive statistics were processed in RStudio and Microsoft Excel. Statistical analysis was performed using RStudio statistics program. Chi-square test of independence was performed on variables to determine if an association exists, with p-value $\leq 0.05$ were considered statistically significant.

\section{RESULTS}

A total of eighty-four consecutive cases of fungal endophthalmitis, that is eighty-four eyes, were included in this single centre case series over the 20-year period. The registry captured an average of 4.8 fungal cases per year. The median age of patients was 43.5 years [IQR $30.8-63.0$ ], and $65.5 \%(n=55)$ of patients were male. Clinical characteristics of patients are listed in Table 1 and Appendix 1.11 (13.1\%) patients had history of type II diabetes; with further 15 (17.9\%) patients had immunosuppression in form of chemotherapy or systemic steroids. 24 (28.6\%) patients had a history of Hepatitis C.

$43(51.2 \%)$ patients showed left eye laterality on presentation. 82 patients had a documented presenting VA. Presenting VA ranged from $6 / 5$ to PL with median VA of 6/120. 9 out of 82 cases (11.0\%) presented with no vision impairment, 8 cases $(9.8 \%)$ presented with mild vision impairment, 23 cases (28.0\%) presented with 
moderate vision impairment, 6 cases (7.3\%) presented with severe vision impairment and 39 cases (47.6\%) were blind on presentation (Table 1 ).

\begin{tabular}{|c|c|c|}
\hline & $n$ & $\%$ \\
\hline Sex - Male & 55 & 65.5 \\
\hline Median age (years) & $\begin{array}{l}43.5[\text { IQR } 30.8- \\
63.0]\end{array}$ & \\
\hline Type II diabetes & 11 & 13.1 \\
\hline Immunosuppression & 15 & 17.9 \\
\hline Hepatitis C & 24 & 28.6 \\
\hline \multicolumn{3}{|l|}{ Presenting Snellen VA } \\
\hline No vision impairment $(\geq 6 / 12)$ & 9 & 11.0 \\
\hline Mild vision impairment ( $>6 / 12$ to $\leq 6 / 18$ ) & 8 & 9.8 \\
\hline $\begin{array}{l}\text { Moderate vision impairment ( }>6 / 18 \text { to } \\
\leq 6 / 60 \text { ) }\end{array}$ & 23 & 28.0 \\
\hline $\begin{array}{l}\text { Severe vision impairment ( }>6 / 60 \text { to } \\
\leq 3 / 60 \text { ) }\end{array}$ & 6 & 7.3 \\
\hline Blind ( $<3 / 60$ to no light perception) & 39 & 47.6 \\
\hline \multicolumn{3}{|l|}{ Final Snellen VA } \\
\hline No vision impairment $(\geq 6 / 12)$ & 31 & 36.9 \\
\hline Mild vision impairment ( $>6 / 12$ to $\leq 6 / 18$ ) & 8 & 9.5 \\
\hline $\begin{array}{l}\text { Moderate vision impairment ( }>6 / 18 \text { to } \\
\leq 6 / 60)\end{array}$ & 13 & 15.5 \\
\hline $\begin{array}{l}\text { Severe vision impairment ( }>6 / 60 \text { to } \\
\leq 3 / 60 \text { ) }\end{array}$ & 1 & 1.2 \\
\hline Blind ( $<3 / 60$ to no light perception) & 14 & 16.7 \\
\hline Enucleation & 4 & 4.8 \\
\hline LFU & 13 & 15.5 \\
\hline LOS (median) & $\begin{array}{l}4.0[\mathrm{IQR} 2.0- \\
6.0]\end{array}$ & \\
\hline
\end{tabular}

VA, visual acuity; LOS, length of stay; LFU, lost to follow-up

An endogenous source was suspected in $68(81.0 \%)$ cases, followed by penetrating eye injury $(n=9,10.7 \%)$ and cataract surgery $(n=3,3.6 \%)$ (Table 2$)$. The source of 
endogenous seeding was mainly attributable to IVDU ( $n=38,55.9 \%)$, followed by other haematogenous source $(n=13,19.1 \%)$, grafts/intravenous lines $(n=7,10.3 \%)$, urinary source $(n=4,5.9 \%)$, bowel $(n=4,5.9 \%)$ and transplants $(n=2,2.9 \%)$. The time to presentation with fungal endophthalmitis was variable for patients with history of IVDU and other endogenous sources due to incomplete history. All cases presenting with PEI were reviewed in the emergency department within 3 to 48 hours. One case presented 370 days post-glaucoma surgery while the mean time to presentation was 78.5 days (range 67 to 90 days) for 2 post-cataract surgery cases and unknown for 1 case. One fungal endophthalmitis case secondary to penetrating keratoplasty presented 4 days post procedure.

\begin{tabular}{|lll|}
\hline \multicolumn{3}{|l|}{ Table 2: Causes of fungal endophthalmitis cases over 20 years $(\mathrm{n}=84)$} \\
\hline Causes & Total $(n)$ & $\%$ \\
\hline Endogenous & 68 & 81.0 \\
PEI & 9 & 10.7 \\
Cataract & 3 & 3.6 \\
Corneal ulceration & 1 & 1.2 \\
Glaucoma related & 1 & 1.2 \\
IVI & 1 & 1.2 \\
Other surgery (penetrating keratoplasty) & 1 & 1.2 \\
\hline
\end{tabular}

$P E I$, penetrating eye injury; IVI, intravitreal injections

79 out of 84 (94.0\%) cases underwent intravitreal acquisition of ocular fluids and administration of intravitreal therapy (tap-and-inject procedure). Repeat injection was performed in $44(52.4 \%)$ cases due to poor clinical response to the initial injection. For initial intravitreal therapy, Amphotericin B and Vancomycin were the most commonly used agents in combination or single agent regimes, followed by Ceftazidime and Voriconazole (Table 3). The median number of tap-and-inject procedures per patient was 2 (Range 0 to 4). The average number of intravitreal anti-fungal agents given during the tap-and-inject procedure was 1.3 (Range 0 to 3). 72 out of $84(85.7 \%)$ patients received systemic antifungal therapy (single agent or 
combined), where 35 (48.6\%) patients received Fluconazole and 15 (20.8\%) patients received Voriconazole. The duration of systemic antifungal therapy was given for a minimum of 4 to 6 weeks in cases with Candida positive endophthalmitis. The duration of therapy in other cases was variable and was determined by the aetiology, microbiology growth, extent and response to systemic antifungal therapy. The exact duration of treatment was difficult to ascertain in certain cases being lost to follow-up.

\begin{tabular}{|lll|}
\hline Table 3: First intravitreal injection $(\mathrm{n}=79)$ & \\
\hline Medication & Total $(\mathrm{n})$ & $\%$ \\
\hline Amphotericin B & 42 & 53.2 \\
Vancomycin & 42 & 53.2 \\
Ceftazidime & 36 & 45.6 \\
Voriconazole & 19 & 24.1 \\
Dexamethasone & 11 & 13.9 \\
Fluconazole & 1 & 1.3 \\
Foscarnet & 1 & 1.3 \\
Teicoplanin & 1 & 1.3 \\
\hline
\end{tabular}

+ Intravitreal dexamethasone was used in cases initially suspected to be secondary to bacterial aetiology. These cases were included in the study as they subsequently grew fungal species in the ocular fluid specimens.

37 (44.0\%) patients underwent pars plana vitrectomy (PPV). 20 (54.1\%) of these procedures were performed during the first 24 hours of their presentation (classified as early) due to severe inflammation on presentation. The remainder of cases 17 (45.9\%) cases underwent vitrectomy after 24 hours (classified as delayed) due to worsening disease. There was no statistically significant correlation between presenting VA (less or severe vision impairment vs. worse or blind) and PPV $\left(X^{2}=\right.$ $0.001, p=0.97$ ). There was also no statistically significant correlation between patient immunosuppression and PPV $\left(X^{2}=0.015, p=0.90\right)$. 
Microbiology analysis showed 55 (65.6\%) cases were culture positive for fungal species (Table 4). 17 out 20 cases with early PPV were culture positive. Remainder of cases ( $n=41$ ) showed fungal growth with vitreous biopsy (tap) alone. All pathology results positive for Candida spp. were sensitive to treatment with Fluconazole. 3 cases, initially managed as fungal endophthalmitis based on examination findings or clinical suspicion, grew bacterial pathogens. One patient cultured Streptococcus salivarius in blood, another cultured Streptococcus oralis from aqueous humour and another patient with history of Human Immunodeficiency Virus infection was positive for Treponema pallidum. There was no growth documented in 26 cases, including some cases with repeat tap-and-inject procedure. In patients with a history of IVDU, 13 out of 38 patients had no growth, 23 cases grew Candida spp., 1 patient isolated Aspergillus spp. and 1 patient isolated Streptococcus salivarius.

\begin{tabular}{|lll|}
\hline \multicolumn{2}{|c|}{ Table 4: Fungal isolates from intraocular fluid samples $(\mathrm{n}=55)$} \\
\hline Genus & Species & Total \\
\hline Candida & albicans & 39 \\
& dubliniensis & 23 \\
& parapsilosis & 6 \\
& tropicalis & 3 \\
& Sake & 5 \\
& Undifferentiated & 1 \\
Aspergillus & & 1 \\
& Undifferentiated & 5 \\
Cryptococcus & terrein & 4 \\
Curvularia & neoformans & 1 \\
& & 1 \\
Gongronella & Iunata & 2 \\
& Undifferentiated & 1 \\
& butleri & 1 \\
\hline
\end{tabular}




\begin{tabular}{|lll|}
\hline Paecilomyces & lilacinus & 1 \\
Scedosporium & & 3 \\
& prolificans & apiospermum \\
Undifferentiated fungi/yeast & & 2 \\
\hline
\end{tabular}

In terms of visual outcomes, $43(51.2 \%)$ of the cases showed improvement in their Snellen VA, while 12 cases ( $14.3 \%$ ) showed no change, 11 cases ( $13.1 \%$ ) deteriorated, and 14 cases (16.7\%) were lost to follow up (Figure 1 ).

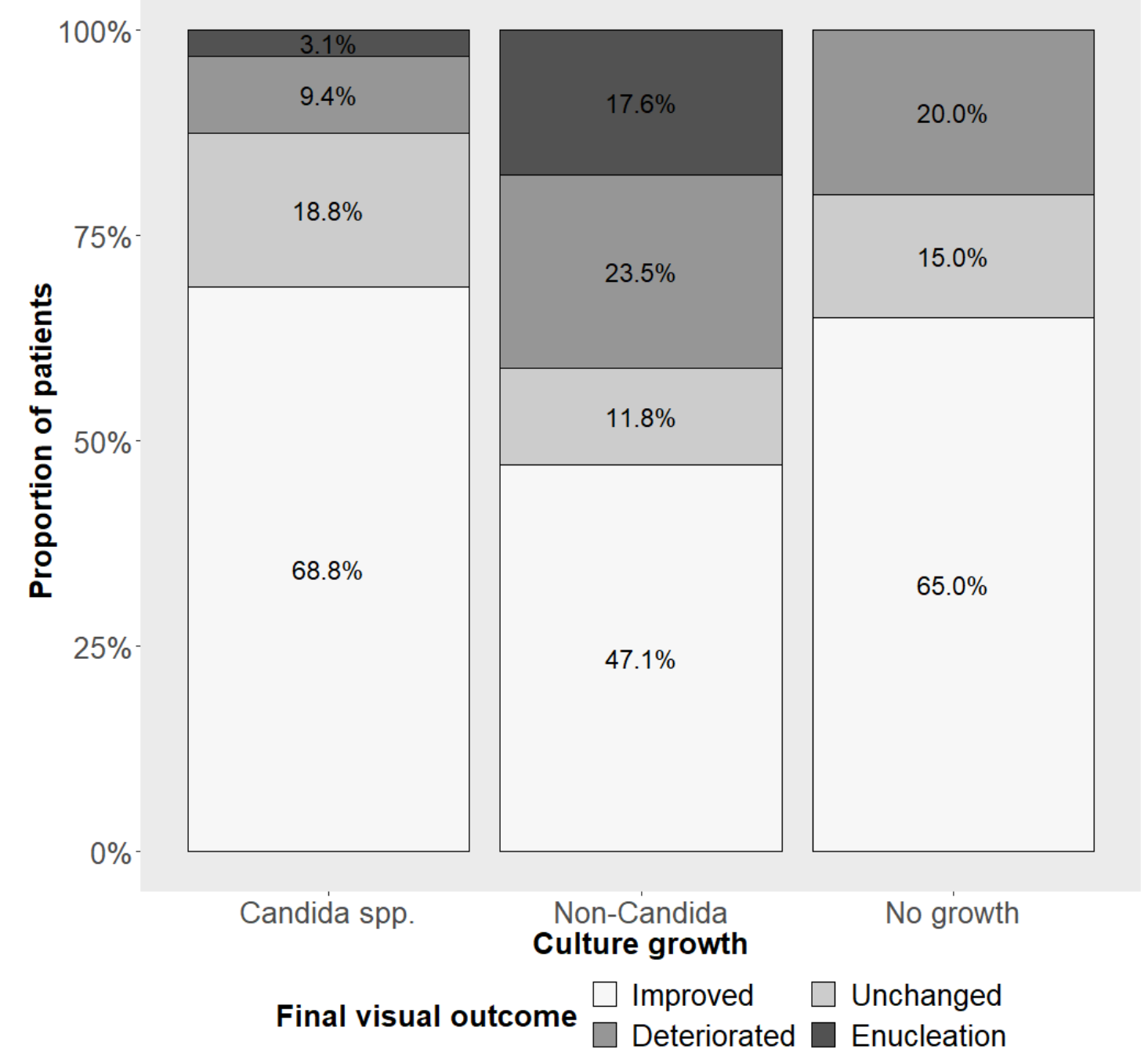

Figure 1: Final visual outcomes and culture growth 
Further breakdown of presenting visual acuity and final visual outcomes according to fungal genus growth is demonstrated in Table 5.

Table 5: Fungal isolates from intraocular fluid samples vs. presenting and final visual acuity $(\mathrm{n}=81)$

\begin{tabular}{|c|c|c|c|c|}
\hline Genus (n) & $\begin{array}{l}\text { Presenting } \\
\text { VA }\end{array}$ & $n$ & Final VA & $n$ \\
\hline \multirow[t]{7}{*}{ Candida (39) } & No & 2 & No & 15 \\
\hline & Mild & 2 & Mild & 7 \\
\hline & Moderate & 14 & Moderate & 4 \\
\hline & Severe & 4 & Severe & 1 \\
\hline & Blind & 16 & Blind & 5 \\
\hline & NA & 1 & Enucleation & 1 \\
\hline & & & LFU & 6 \\
\hline \multirow[t]{3}{*}{ Aspergillus (5) } & Moderate & 1 & Moderate & 2 \\
\hline & Blind & 4 & Blind & 1 \\
\hline & & & Enucleation & 2 \\
\hline Cryptococcus (1) & Blind & 1 & Moderate & 1 \\
\hline \multirow[t]{2}{*}{ Curvularia (2) } & No & 1 & No & 1 \\
\hline & Blind & 1 & Blind & 1 \\
\hline Gongronella (1) & Moderate & 1 & No & 1 \\
\hline Paecilomyces (1) & Blind & 1 & Moderate & 1 \\
\hline \multirow[t]{3}{*}{ Scedosporium (3) } & Moderate & 1 & Blind & 1 \\
\hline & Blind & 2 & Enucleation & 1 \\
\hline & & & LFU & 1 \\
\hline \multirow{3}{*}{$\begin{array}{l}\text { Undifferentiated } \\
\text { fungi/yeast ( } 3 \text { ) }\end{array}$} & No & 1 & No & 2 \\
\hline & Moderate & 1 & Blind & 1 \\
\hline & Blind & 1 & & \\
\hline \multirow[t]{6}{*}{ No growth (26) } & No & 4 & No & 10 \\
\hline & Mild & 4 & Mild & 1 \\
\hline & Moderate & 3 & Moderate & 4 \\
\hline & Severe & 2 & Blind & 5 \\
\hline & Blind & 12 & LFU & 6 \\
\hline & NA & 1 & & \\
\hline
\end{tabular}

VA, visual acuity; No vision impairment ( $\geq 6 / 12)$; Mild vision impairment ( $>6 / 12$ to $\leq 6 / 18)$; Moderate vision impairment ( $>6 / 18$ to $\leq 6 / 60)$; Severe vision impairment 
(>6/60 to S3/60); Blind (<3/60 to no light perception); LFU, lost to follow-up; $N A$, not available.

There was no statistically significant correlation between patients undergoing PPV and improved visual outcomes $\left(X^{2}=0.278, p=0.60\right)$. The 4 cases that underwent enucleation were culture positive for Aspergillus spp. $(n=2)$, Candida albicans $(n=1)$ and Scedosporium prolificans $(n=1)$. There was no significant difference between Candida or non-Candida growth or no growth and presence of improvement in visual outcome $\left(x^{2}=2.311\right.$, df $\left.=2, p=0.32\right)$.

\section{DISCUSSION}

Our study outlines the presentation of clinically diagnosed and/or microbiologically proven fungal endophthalmitis cases over twenty years at a tertiary referral centre for ocular emergencies. Our data shows that the majority of patients with fungal endophthalmitis were secondary to an endogenous source, with IVDU being the most common cause, comprising 55.9\% $(n=38)$ of these cases. Similarly, case series from centres in South Australia and Florida have reported a high proportion of fungal endophthalmitis cases secondary to IVDU. ${ }^{19,20}$ There has been a steady increase of IVDU related cases observed in developed countries when compared to the data from the developing world. ${ }^{8,18}$ Fewer cases of fungal endogenous endophthalmitis have been reported in population studies from China, India and Korea. ${ }^{21,22,23}$ Conversely, a low number of fungal endophthalmitis secondary to PEI $(n=9,10.7 \%)$ was seen in our study group. In a 14-year review of patients from a centre in India, Chakrabarti et al demonstrated a high incidence of fungal endophthalmitis attributed to post-trauma cases. ${ }^{10}$ 
As a result, the causative organisms in our population were dissimilar to those seen in India and China. ${ }^{8,27}$ Aspergillus spp. has been commonly reported in those fungal endophthalmitis cohorts while our series shows higher number of Candida spp. growth. This is likely secondary to the causative mechanisms attributable to fungal endophthalmitis. Yeasts have been commonly associated with endogenous fungal endophthalmitis (especially in cases with IVDU), while pathogens related to exogenous fungal endophthalmitis are variable. Higher rates of evisceration and poor visual outcomes have been reported in cases with Aspergillus spp. growth. ${ }^{20,29}$ Some of the risk factors associated with enucleation/evisceration include endophthalmitis secondary to corneal ulceration or endogenous endophthalmitis and initial poor visual acuity. ${ }^{30}$ This is reflected in our case series but the number of cases ( $n=4,4.5 \%$ ) undergoing enucleation was low. Two out of the four cases grew Aspergillus spp. The range of presenting Snellen VAs for these patients ranged from $6 / 24$ to HM. Additionally, two patients were immunosuppressed and one patient had a penetrating eye injury.

In an Indian population, a high proportion of fungal endophthalmitis was reported in post-cataract surgery patients, ${ }^{24}$ while a review by Smith et al demonstrated a lower proportion of fungal endophthalmitis attributable to cataract surgery in a Western population. ${ }^{25}$ Patient factors associated with this include immunocompromised status, pre-operative fungal keratitis, or operative factors like contamination of intraocular fluid with irrigation solutions used during surgery or environmental factors. ${ }^{25}$ This lower proportion of post-cataract surgery fungal endophthalmitis is reflected in our dataset; only 3 out of 84 (3.6\%) cases were attributable to cataract surgery. One patient had a history of diabetes mellitus but remainder of the cases had no identifiable immunocompromise. 
$69.0 \%$ culture positivity rate in our study is higher when compared to global standards. Two large endophthalmitis cohort studies over 14 and 25 years demonstrated a lower culture positivity rate in Indian populations when compared to our results. ${ }^{10,27}$ Similarly, a lower culture positivity rate of $51 \%$ in blood samples and $48 \%$ from vitreous samples has been described in Danish population with endogenous endophthalmitis. ${ }^{39}$ These differences seen may be secondary to microbiology analysis techniques used at each of these centres.

A large proportion of our study population received systemic therapy with Fluconazole and Voriconazole. Fluconazole and Voriconazole - intravitreally or systemically - remain a safe choice for treatment of fungal endophthalmitis due to excellent intraocular concentrations and safety. ${ }^{28}$ Intravenous Amphotericin B carries risk of nephrotoxicity and infusion related toxicity, along with poor intraocular penetration. ${ }^{2,28}$ Amphotericin B was the treatment of choice for first intravitreal injection up until 2010, where the standard treatment changed to intravitreal Voriconazole in our study population.

The role of pars plana vitrectomy (PPV) in fungal endophthalmitis remains controversial. PPV may aid diagnosis by providing a better sample quality; reduce the fungal load from the intraocular structures, improve retinal oxygenation; reduce the incidence and severity of retinal complications, especially macular complications. Early PPV has prophylactic benefits in reduction of the duration of disease processes. ${ }^{40} \mathrm{~A}$ small number of case series have reported improvement in visual outcomes following PPV. 1,2,12 We did not find an association between patients undergoing PPV and final visual outcome. Furthermore, it is uncertain if the time from presentation to PPV has an impact on visual outcomes. Studies examining the difference between early ( $<24$ hours from presentation) and delayed ( $>24$ hours) PPV are limited in fungal endophthalmitis populations due to small sample sizes. 
Only 20/37 of our cases underwent early PPV. The sample size was too small to comment on any difference between the groups. Although vitrectomy has not been proven to directly improve visual outcomes, PPV remains a useful diagnostic tool. Our case series showed initial pathogen isolation rate with vitreous biopsy was $48.8 \%$ compared to $85 \%$ with early PPV. PPV is also useful in the management of post-infection sequelae such as retinal detachment, epiretinal membrane, choroidal neovascular membrane, and macular hole formation. [2]

There is an important limitation to this study. Whilst an endophthalmitis management guideline is in place, given the nature of fungal endophthalmitis clinician discretion plays a major role in management of these cases, resulting in variation in treatment.

In conclusion, whilst fungal endophthalmitis is a diagnostic and management challenge with potential for significant long-term visual impairment, there is scope for improvement in patient outcomes. Due to a large of proportion of cases attributable to IVDU in our group, a thorough drug history and a higher index of suspicion of fungal endophthalmitis amongst these patients is recommended. Secondly, there is a wide range of causative organisms, most frequently involving Candida spp., which were all sensitive to Fluconazole from our population. Hence, empirical treatment with Fluconazole might be beneficial in suspected cases to reduce pathogen load promptly (Figure 2). Lastly, vitrectomy is a useful diagnostic tool with high pathogen isolation rate, however its role in improvement of visual outcomes needs to be further examined. 


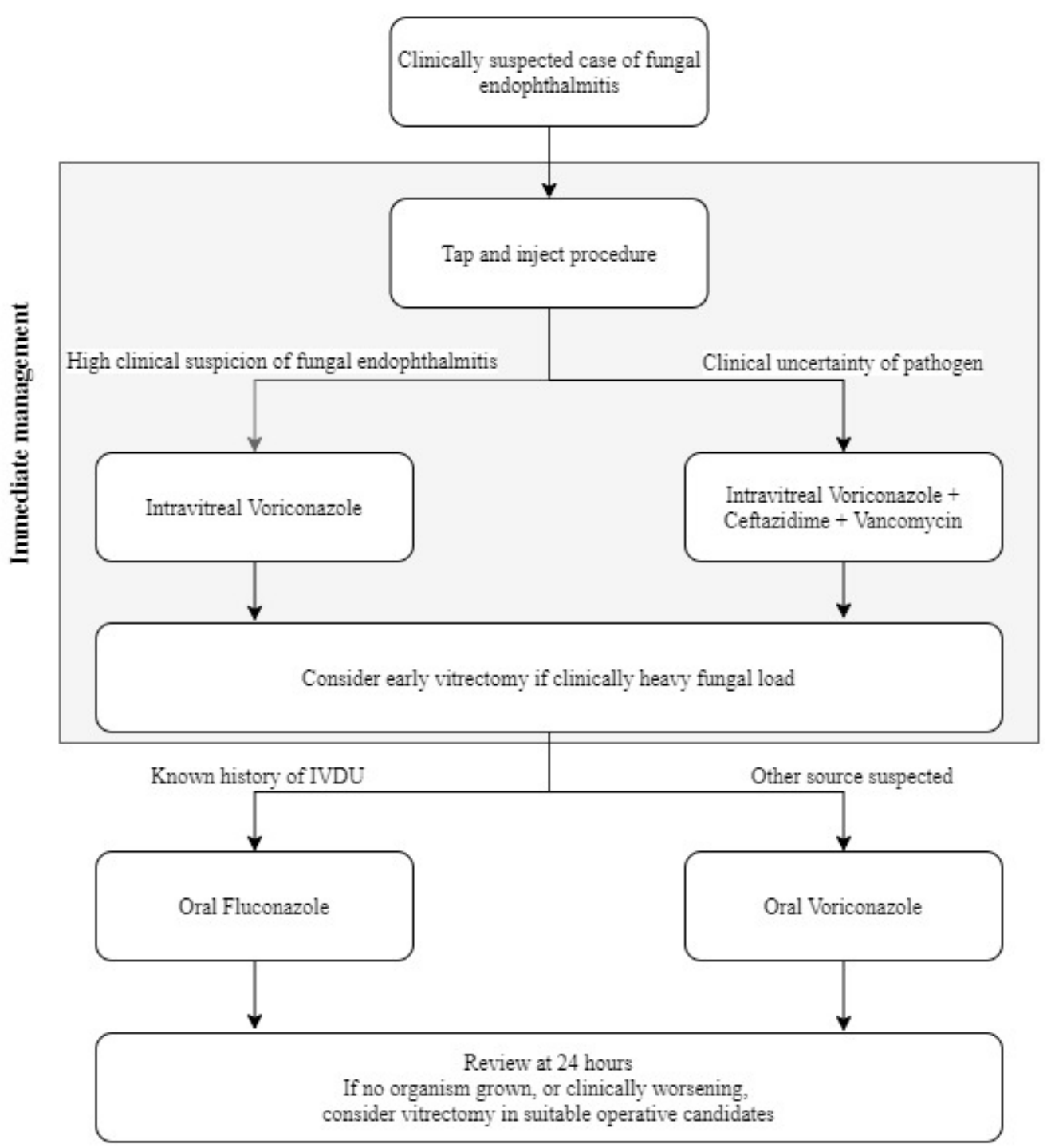

Figure 2: Protocol for management of patient with suspected fungal endophthalmitis

\section{ACKNOWLEDGEMENTS}

Study data was collected and managed using REDCap electronic data capture tools hosted at the Centre for Eye Research Australia. The Centre of Eye Research Australia receives operational infrastructure support from the Victorian Government. 


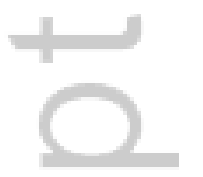




\section{REFERENCES}

1. Celiker $\mathrm{H}$, Kazokoglu $\mathrm{H}$. The role of pars plana vitrectomy in the management of fungal endogenous endophthalmitis. Eur J. Ophthalmol. 2018 Nov 26: 1120672118815105. doi: 10.1177/1120672118815105.

2. Chee YE, Eliott D. The Role of Vitrectomy in the Management of Fungal Endophthalmitis. Semin Ophthalmol. 2017;32(1):29-35; doi: 10.1080/08820538.2016.1228396.

3. Teng YT, Teng MC, Kuo HK, Fang PC, Wu PC, Chen CH et al. Isolates and antibiotic susceptibilities of endophthalmitis in postcataract surgery: a 12 year-review of culture-proven cases. Int Ophthalmol. 2017 J un;37(3):513518. doi: 10.1007/s10792-016-0288-2.

4. Sadiq MA, Hassan M, Agarwal A, et al. Endogenous endophthalmitis: diagnosis, management, and prognosis. J Ophthalmic Inflamm Infect. 2015;5: 32. doi: 10.1186/s12348-015-0063-y

5. Kostick DA, Foster RE, Lowder CY, Meyers SM, McHenry MC. Endogenous endophthalmitis caused by Candida albicans in a healthy woman. $\mathrm{Am} \mathrm{I}$ Ophthalmol. 1992 May 15;113(5):593-5. doi:10.1016/s0002-9394(14)74741-5

6. Valluri S, Moorthy RS, Liggett PE, Rao NA. Endogenous Aspergillus endophthalmitis in an immunocompetent individual. Int Ophthalmol. 1993 Jun;17(3):131-5.

7. Shankar K, Gyanendra L, Hari S, Narayan SD. Culture proven endogenous bacterial endophthalmitis in apparently healthy individuals. Ocul Immunol Inflamm. 2009 Nov-Dec;17(6):396-9. doi: 10.3109/09273940903216891.

8. Connell PP, O'Neill EC, Amirul Islam FM, Buttery R, McCombe M, Essex RH, Roufail E, Lash S, Wolffe B, Clark B, Chiu D, Campbell W, Allen P. Endogenous endophthalmitis associated with intravenous drug abuse: seven-year experience at a tertiary referral center. Retina 2010 Nov-Dec;30(10):1721-5. 
9. Takebayashi K, Matsumoto S, Nakagawa Y, Wakabayashi S, Aso Y, Inukai T. Endogenous endophthalmitis and dissemi- nated intravascular coagulation complicating a Klebsiella pneumoniae perirenal abscess in a patient with type 2 diabetes. AmJ Med Sci 2005;329:157-160.

10. Chakrabarti A, Shivaprakash MR, Singh R, Tarai B, George VK, Fomda BA, Gupta A. Fungal endophthalmitis: fourteen years' experience from a centre in India. Retina 2008 Nov-Dec; 28(10): 1400-7.

11. Connell PP, O'Neill EC, Fabinyi D et al. Endogenous endophthalmitis: 10-year experience at a tertiary referral centre. Eye(Lond). 2011 Jan; 25(1):66-72. doi: 10.1038/eye.2010.145

12. Lingappan A, Wykoff CC, Albini TA, et al. Endogenous fungal endophthalmitis: Causative organisms, management strategies, and visual acuity outcomes. AmJ Ophthalmol. 2012;153(1):162-6.e1. doi:10.1016/j.ajo.2011.06.020.

13. Essman TF, Flynn HWJ, Smiddy WE, et al. Treatment outcomes in a 10-year study of endogenous fungal endophthalmitis. Ophthalmic Surg Lasers. 1997;28(3):185-194.

14. Wykoff CC, Flynn HW J r, Miller D, Scott IU, Alfonso EC. Exogenous fungal endophthalmitis: microbiology and clinical outcomes. Ophthalmology 2008 Sep:115(9):1501-7.

15. Gupta S, Loudill C, Tammara A, Chow RT. A rare case of bilateral aspergillus endophthalmitis. J Community Hosp Intern Med Perspect. 2015;5(6):28984.

16. Gross J G. Endogenous Aspergillus-induced endophthalmi- tis: Successful treatment without systemic antifungal medication. Retina (Philadelphia, PA). 1992;12(4):341-345.

17. World Health Organization International Statistical Classification of Diseases and Related Health Problems 10th Edition Chapter VII H54 Blindness and low vision. http://www. who. int/classifications/icd/en/ 
18. Relhan N, Schwartz SG, Flynn HW Jr. Endogenous fungal endophthalmitis: An Increasing Problem Among Intravenous Drug Users. JAMA. 2017 Aug 22;318(8):741-742. doi: 10.1001/jama.2017.10585.

19. Leibovitch I, Lai T, Raymond G, Zadeh R, Nathan F, Selva D. Endogenous endophthalmitis: a 13-year review at a tertiary hospital in South Australia. Scand J Infect Dis. 2005;37(3):184-9.

20. Schiedler V, Scott IU, Flynn HW J r., Davis J L, Benz MS, Miller D. Cultureproven endogenous endophthalmitis: clinical features and visual acuity outcomes. AmJ Ophthalmol. 2004 Apr; 137(4): 725-31

21. Duan F, Wu K, Liao J, Zheng Y, Yuan Z, Tan J, Lin X. Causative Microorganisms of Infectious Endophthalmitis: A 5-Year Retrospective Study. J Ophthalmol. 2016;2016: 6764192. doi: 10.1155/2016/6764192.

22. Sharma S, Padhi TR, Basu S, Kar S, Roy A, Das T. Endophthalmitis patients seen in a tertiary eye care centre in Odisha: a clinico-microbiological analysis. Indian J Med Res. 2014 Jan; 139(1): 91-8.

23. Lim HW, Shin JW, Cho HY et al. Endogenous endophthalmitis in the Korean population: a six-year retrospective study. Retina. 2014 Mar;34(3):592-602. doi: 10.1097/IAE.0b013e3182a2e705

24. Jindal A, Pathengay A, J alali S, et al. Microbiologic spectrum and susceptibility of isolates in delayed post-cataract surgery endophthalmitis. Clin Ophthalmol. 2015; 9: 1077 - 1079. doi: 10.2147/OPTH.S82852

25. Smith TC, Benefield RJ, Kim JH. Risk of Fungal Endophthalmitis Associated with Cataract Surgery: A Mini-Review. Mycopathologia. 2015 Dec; 180(56):291-7. doi 10.1007/s11046-015-9932-z. Epub 2015 Aug 29.

26. Sowmya $P$, Madhavan HN. Diagnostic utility of polymerase chain reaction on intraocular specimens to establish the etiology of infectious endophthalmitis. Eur J Ophthalmol 2009; 19: 812-817.

27.J oseph J, Sontam B, Guda SJ M et al. Trends in microbiological spectrum of 
endophthalmitis at a single tertiary care ophthalmic hospital in India: a review of 25 years. Eye (Lond). 2019 J uly; 33(7):1090-1095. doi: 10.1038/s41433019-0380-8

28. Riddell IV J, Comer GM, Kauffman CA. Treatment of Endogenous Fungal Endophthalmitis: Focus on New Antifungal Agents. Clin Infect Dis 2011;52(5):648-53. doi: 10.1093/cid/ciq204.

29. Dave TV, Dave VP, Sharma S, Karolia R, J oseph J, Pathengay A et al. Infectious endophthalmitis leading to evisceration: spectrum of bacterial and fungal pathogens and antibacterial susceptibility profile. J Ophthalmic Inflamm Infect. 2019 May 16; 9(1):9. doi: 10.1186/s12348-019-0174-y.

30. Lu X, Ng DS, Zheng K, Peng K, J in C, Xia H et al. Risk factors for endophthalmitis requiring evisceration or enucleation. Sci Rep. 2016 J un 15;6:28100. doi: 10.1038/srep28100.

31. Mithal K, Pathengay A, Bawdekar A, et al. Filamentous fungal endophthalmitis: results of combination therapy with intravitreal amphotericin B and voriconazole. Clin. Ophthalmol. 2015; 9: 649 - 655. doi:

\subsection{7/OPTH.S80387}

32. Tirpack AR, Duker JS, Baumal CR. An Outbreak of Endogenous Fungal Endophthalmitis Among Intravenous Drug Abusers in New England. JAMA Ophthalmol. 2017 J un 1; 135(6)534:540. doi: 10.1001/jamaophthalmol.2017.0650.

33. Behera UC, Budhwani M, Das T et al. Role of Early Vitrectomy in the Treatment of Fungal Endophthalmitis. Retina. 2018 J ul; 38(7): 1385-1392. doi: 10.1097/IAE.0000000000001727.

34. World Health Organization International Statistical Classification of Diseases and Related Health Problems 10th Edition Chapter VII H54 Blindness and low vision. http://www. who.int/classifications/icd/en/

35. Celiker H, Kazokoglu H. Ocular culture-proven endogenous endophthalmitis: a 
5-year retrospective study of the microorganism spectrum at a tertiary referral center in Turkey. Int Ophthalmol. 2019 Aug; 39(8):1743-1751. doi: 10.1007/s10792-018-0997-9.

36. Liu MY, Zhang L, Yin XL, Sun SY. Endophthalmitis associated with fungal keratitis and penetrating injuries in North China. Eur J Ophthalmol. 2019 Mar 4: :1120672119833896. doi: 10.1177/1120672119833896.

37. Durand ML. Endophthalmitis. Clin Microbial Infect 2013 Mar:19(3):227-34.

38. Aboltins CA, Allen P, Daffy JR. Fungal endophthalmitis in intravenous drug users injecting buprenorphine contaminated with oral Candida species. Med J Aust 2005; 182: 427.

39. Bjerrum SS, la Cour M. 59 eyes with endogenous endophthalmitis- causes, outcomes and mortality in a Danish population between 2000 and 2016. Graefes Arch Clin Exp Ophthalmol. 2017 Oct;255(10):2023-2027. doi: 10.1007/s00417-017-3760-4.

40. Kirchhof B., Wong D. Vitreo-retinal Surgery. Essentials in Ophthalmology. Berlin, Heidelberg: Springer; 2007. 5: Complete and Early Vitrectomy for Endophthalmitis (CEVE) as Today's Alternative to the Endophthalmitis Vitrectomy Study; 53-68pp. 


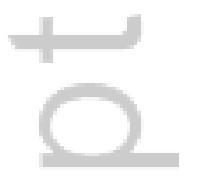




\section{University Library}

\section{- M M N E R VA A gateway to Melbourne's research publications}

Minerva Access is the Institutional Repository of The University of Melbourne

Author/s:

Bhullar, GK;Dawkins, RCH;Paul, RA;Allen, PJ

Title:

Fungal endophthalmitis: A 20-year experience at a tertiary referral centre

Date:

2020-08-20

Citation:

Bhullar, G. K., Dawkins, R. C. H., Paul, R. A. \& Allen, P. J. (2020). Fungal endophthalmitis:

A 20-year experience at a tertiary referral centre. CLINICAL AND EXPERIMENTAL OPHTHALMOLOGY, 48 (7), pp.964-972. https://doi.org/10.1111/ceo.13820.

Persistent Link:

http://hdl.handle.net/11343/276175 